\title{
ON PROJECTIVE CHARACTERS OF THE SAME DEGREE
}

\author{
by R. J. HIGGS
}

(Received 15 January, 1997)

0. Introduction. All groups $G$ considered in this paper are finite and all representations of $G$ are defined over the field of complex numbers. The reader unfamiliar with projective representations is referred to [9] for basic definitions and elementary results.

Let $\operatorname{Proj}(G, \alpha)$ denote the set of irreducible projective characters of a group $G$ with cocyle $\alpha$. In previous papers (for exampe [2], [4], and [6]) numerous authors have considered the situation when $|\operatorname{Proj}(G, \alpha)|=1$ or 2 ; such groups are said to be of $\alpha$-central type or of $2 \alpha$ central type, respectively. In particular in [4, Theorem A] the author showed that if $\operatorname{Proj}(G, \alpha)=\left\{\xi_{1}, \xi_{2}\right\}$, then $\xi_{1}(1)=\xi_{2}(1)$. This result has recently been independently confirmed in $[8$, Corollary $C]$.

The aim of this short paper is to provide some positive evidence about the following conjecture, of which the result mentioned above is just a special case.

CONJECTURE. Let $G$ be a group and $\alpha$ be a cocycle of $G$. Then either $G$ is of $\alpha$-central type or $\operatorname{Proj}(G, \alpha)$ contains at least two elements of the same degree.

The reader will discover that groups of $\alpha$-central type play an important part in our investigation of the conjecture, which we are able to verify in a number of cases; most notably when $G$ is supersoluble or has odd order. trivial.

1. Characters of the smallest degree. We start by considering the situation when $\alpha$ is

Lemma 1.1. Let $G$ be a non-trivial group. Then $\operatorname{Irr}(G)$ do not all have different degrees.

Proof. Let $G$ be a counterexample of minimal order. Suppose $N$ is a proper normal subgroup of $G$. Then $\operatorname{Irr}(G / N)$ contains two elements of the same degree, which lift irreducibly to $G$. So $G$ must be a non-abelian simple group, and moreover all of its irreducible charcaters must be rational valued. Thus $G \cong S p_{6}$ (2) or $O_{8}^{+}(2)^{\prime}$ from [3, Corollary B.1], but from [1] both these groups do possess irreducible characters of the same degree.

As a consequence of Lemma 1.1, we can assume henceforward where necessary that $o([\alpha])>1$ in $\mathrm{M}(G)$, the Schur multiplier of $G$. We now proceed to verify the conjecture in a number of easy cases, these cases have in common the fact that we need only to consider irreducible projective characters of the smallest degree. To avoid repetition $\alpha$ will always denote a cocycle of the group $G$ under consideration in the following results.

Lemma 1.2. Let $G$ be a p-group. Then either $G$ is of $\alpha$-central type or $\operatorname{Proj}(G, \alpha)$ contains $n$ elements of the smallest degree where $n \equiv 0(\bmod p)$.

Glasgow Math. J. 40 (1998) 431-434. 

Then,

Proof. Let $\operatorname{Proj}(G, \alpha)=\left\{\xi_{1}, \ldots, \xi_{t}\right\}$, with $\xi_{1}$ being an element of the smallest degree.

$$
|G| /\left(\xi_{1}(1)\right)^{2}=\sum_{i=1}^{t}\left(\xi_{i}(1) / \xi_{1}(1)\right)^{2}
$$

Now $G$ is of $\alpha$-central type if and only if $t=1$. If $t>1$ the left hand side of the above equation is congruent to 0 modulo $p$, so there must be $n$ elements $\xi_{i} \in \operatorname{Proj}(G, \alpha)$ with $n \equiv 0(\bmod p)$ such that $\xi_{i}(1)=\xi_{1}(1)$.

COROLlaRY 1.3. Let $G$ be a nilpotent group and $\left\{p_{i}: 1 \leq i \leq r\right\}$ be the distinct prime divisors of $|G|$. Then either $G$ is of $\alpha$-central type or $\operatorname{Proj}(G, \alpha)$ contains $n$ elements of the smallest degree where $n \equiv 0\left(\bmod p_{i}\right)$ for some $i$ with $1 \leq i \leq r$.

Proof. Let $S_{i}$ be the Sylow $p_{i}$-subgroup of $G$. Then it follows from either Corollary 5.1.3 or Theorem 7.1.13 of [9] that there exist cocycles $\alpha_{i}$ of $S_{i}$ such that $\operatorname{Proj}(G, \alpha)=\left\{\lambda\left(\xi_{1} \times \cdots \times \xi_{r}\right): \xi_{i} \in \operatorname{Proj}\left(S_{i}, \alpha_{i}\right)\right\}$, where $\lambda$ is a function from $G$ into the nonzero complex numbers with $\lambda(1)=1$. The result is now immediate from Lemma 1.2.

Our next result covers the case of a metacyclic group.

LeMmA 1.4. Let $G$ be a group, and suppose $G$ contains a normal abelian subgroup $N$ such that $\left[\alpha_{N}\right]=[1]$ and $G / N$ is cyclic. Then either $G$ is of $\alpha$-central type or $\operatorname{Proj}(G, \alpha)$ contains at least two elements of the smallest degree.

Proof. Let $\xi \in \operatorname{Proj}(G, \alpha)$, then $\xi(1)$ divides $[G: N]$ by [11, Theorem 2]. Now assume $\xi$ is of the smallest degree, let $\lambda$ be an irreducible constituent of $\xi_{N}$, and $I$ denote the inertia subgroup $I_{G}(\lambda)$. Then $\lambda(1)=1$, since $N$ is abelian and $\left[\alpha_{N}\right]$ is trivial. Also since $G / N$ is cyclic, the elements of $\operatorname{Proj}(I / N, \beta)$ all have degree one for any cocycle $\beta$ of $I / N$. It follows from the bijections of Clifford's theorem (described in the proof of Theorem 2.1 below), that the $[I: N]$ distinct elements of $\operatorname{Proj}(G, \alpha)$ which are the constituents of $\lambda^{G}$ all have degree $\xi(1)$. We have thus constructed at least two elements of the smallest degree unless $I=N$, and $\lambda^{G}=\xi$. In this case $\xi(1)=[G: N]$, and consequently every element of $\operatorname{Proj}(G, \alpha)$ has this degree. Once again we have at least two elements of the only degree unless $\xi$ is unique and $[G: N]=|N|$.

Our final result in this section has an almost identical proof to Lemma 1.4 , and so the proof is omitted.

Corollary 1.5. Let $G$ be a group, and suppose $G$ contains a normal subgroup $N$ with $\zeta \in \operatorname{Proj}\left(N, \alpha_{N}\right)$ such that $I_{G}(\zeta) / N$ is a non-trivial cyclic group. Then there are at least two elements of $\operatorname{Proj}(G, \alpha)$ of the same degree which are constituents of $\zeta^{G}$.

\section{Supersoluble groups and groups of odd order.}

TheOREM 2.1. Let $G$ be a supersoluble group. Then either $G$ is of $\alpha$-central type or $\operatorname{Proj}(G, \alpha)$ contains at least two elements of the same degree. 
Proof. Let $G$ be a counterexample of minimal order. Let $N$ be a non-trivial normal subgroup of $G$, and $\zeta \in \operatorname{Proj}\left(N, \alpha_{N}\right)$. Let $I=I_{G}(\zeta)$, and $\operatorname{Proj}\left(I \mid \zeta, \alpha_{I}\right)$ denote the set of irreducible constituents of $\zeta^{I}$. Then by [9, Theorem 7.8.10] there exists a cocycle $\beta$ of $I / N$ and bijections $\operatorname{Proj}(I / N, \beta) \rightarrow \operatorname{Proj}\left(I \mid \zeta, \alpha_{l}\right) \rightarrow \operatorname{Proj}(G \mid \zeta, \alpha)$ defined by $\gamma \mapsto \gamma \kappa \mapsto(\gamma \kappa)^{G}$, where $\kappa_{N}=\zeta$ and $\kappa \in \operatorname{Proj}\left(I, \beta^{-1} \alpha_{l}\right)$. The cocycle $\beta^{-1}$ is called an obstruction cocycle, since it obstructs the extension of $\zeta$ to an element of $\operatorname{Proj}\left(I, \alpha_{I}\right)$. Now since $|I / N|<|G|$, either $\operatorname{Proj}(I / N, \beta)$ contains at least two elements of the same degree or $I / N$ is of $\beta$-central type. In the former case the bijections above yield at least two elements of $\operatorname{Proj}(G \mid \zeta, \alpha)$ of the same degree, contrary to the assumption that $G$ is a counterexample. So we must assume $I / N$ is of $\beta$-central type. Consequently $\xi(x)=0$ for all $\xi \in \operatorname{Proj}(G, \alpha)$, and all $x \notin N$. Since $G$ is not of $\alpha$-central type, it must contain a unique minimal normal subgroup $K=\langle x: x$ is $\alpha$-regular $\rangle$.

Since $|K|=p$ for some prime $p, K$ consists of the $\alpha$-regular elements of $G$. Let $S$ be a Sylow $p$-subgroup of $G$. Then $K \leq Z(S)$, so that $S \leq I_{G}(\lambda)$ for all $\lambda \in \operatorname{Proj}\left(K, \alpha_{K}\right)$. Let $H$ be a Hall $p^{\prime}$-subgroup of $G$. Then $H \leq I_{G}(\lambda)$ for some $\lambda \in \operatorname{Proj}\left(K, \alpha_{K}\right)$ by [5, Proposition 1.5 and Corollary 2.4]. It follows from the bijections above that exactly one element $\delta$ of $\operatorname{Proj}\left(K, \alpha_{K}\right)$ is $G$-invariant, and there is a unique $\xi \in \operatorname{Proj}(G \mid \delta, \alpha)$ with $\xi_{K}=e \delta$ and $e^{2}=[G: K]$. Now $\operatorname{Proj}\left(K, \alpha_{K}\right)=\{\delta v: v \in \operatorname{Irr}(K)\}$. Let $v$ be a non-trivial element of $\operatorname{Irr}(K)$, so that $v$ is faithful. Then $I_{G}(\delta v)=I_{G}(v)=C_{G}(K) \triangleleft G$. Thus the $G$-orbits on $\{\delta v: v \neq 1\}$ all have the same length, and for each such orbit we obtain from the bijections above $\xi \in \operatorname{Proj}(G, \alpha)$ with $\xi(1)^{2}=[G: K]\left[G: C_{G}(K)\right]$. Thus there must be a unique such orbit. This implies that $G$ is of $2 \alpha$-central type, contrary to $[4$, Theorem A].

THEOREM 2.2. Let $G$ be a group of odd order. Then either $G$ is of $\alpha$-central type or Proj $(G, \alpha)$ contains at least two elements of the same degree.

Proof. Let $G$ be a counterexample of minimal order. Then the results of the first paragraph of the proof of Theorem 2.1 still hold, and in particular $G$ must contain a unique minimal normal subgroup $K=\langle x: x$ is $\alpha$-regular $\rangle$. Moreover $K$ is abelian since $G$ has odd order. Now if $K \leq Z(G)$, then $\operatorname{Proj}(G, \alpha)$ consists of $|K|$ elements of degree $[G: K]^{1 / 2}$, a contradiction. It follows from [7, Theorem 2.7(b)] that either $K$ is of $\alpha_{K}$-central type or $\left[\alpha_{K}\right]=[1]$. In the former case we obtain that $G$ is of $\alpha$-central type, a contradiction. So $\left[\alpha_{K}\right]=[1]$.

Our argument now follows that of the proof of Theorem A of [4]. Let $C=C_{G}(K)$, and $V=\operatorname{Irr}(K)$. Let $\bar{R}=R / C$ be a chief factor of $G$. Then $\bar{R}$ acts faithfully on $V$ and $C_{V}(\bar{R})$ is trivial, so that $\bar{R}$ has order coprime to $p$. Thus we may use the arguments in the proofs of Lemmas 2.4 and 2.5 of [10] to show that some $\delta \in \operatorname{Proj}(G, \alpha)$ is $G$-invariant. Let $v$ be a nontrivial element of $V$, then $I_{G}(\delta v)=I_{G}(v)=I_{G}\left(v^{-1}\right)=I_{G}\left(\delta v^{-1}\right)$. However since $G$ has odd order $v$ and $v^{-1}$ are not conjugate, and so $\delta v$ and $\delta v^{-1}$ lie in two different orbits of the same length. It follows from the bijections in the proof of Theorem 2.1 that if $\xi_{1}$ is an irreducible constituent of $(\delta v)^{G}$ and $\xi_{2}$ is an irreducible constituent of $\left(\delta v^{-1}\right)^{G}$, then $\xi_{1}(1)=\xi_{2}(1)$, a contradiction.

If the conjecture is true in general then it has the following immediate application to ordinary character theory.

Propositrion 2.3 (Modulo Conjecture). Let $G$ be a group, $N$ be a normal sub group of $G$, and $\vartheta \in \operatorname{Irr}(N)$. Then either $\vartheta^{G}$ has at least two irreducible constituents of the same degree, or each irreducible constituent of $\vartheta^{G}$ vanishes on $G-N$. 
Proof. Let $I=I_{G}(\vartheta)$ and $\beta^{-1}$ denote the cocycle of $I / N$ which obstructs the extension of $\vartheta$ to an element of $\operatorname{Irr}(I)$. Then assuming the conjecture holds either Proj $(I / N, \beta)$ contains at least two elements of the same degree or $I / N$ is of $\beta$-central type. In the former case the bijections in the proof of Theorem 2.1 yield at least two elements of $\operatorname{Irr}(G \mid \vartheta)$ of the same degree. In the latter case using the notation of Theorem $2.1, \operatorname{Irr}(G \mid \vartheta)=\left\{(\gamma \kappa)^{G}\right\}$, where $\gamma$ is the unique element of $\operatorname{Proj}(I / N, \beta)$. Consequently $(\gamma \kappa)^{G}(x)=0$ for all $x \notin N$.

ACKNOWLEDGEMENT. My thanks to the referee for making a number of suggestions which have made this paper more readable.

\section{REFERENCES}

1. J. H. Conway et al., An atlas of finite groups (Oxford University Press, 1985).

2. F. R. DeMeyer and G. J. Janusz, Finite groups with an irreducible representation of large degree, Math. Z. 108 (1969), 145-153.

3. W. Feit and G. M. Seitz, On finite rational groups and related topics, Illinois J. Math. 33 (1988), 103-131. $5-14$.

4. R. J. Higgs, Groups with two projective characters, Math. Proc. Camb. Phil. Soc. 103 (1988),

5. R. J. Higgs, Projective characters of degree one and the inflation-restriction sequence, $J$. Austral. Math. Soc. (Series A) 46 (1989), 272-280.

6. R. B. Howlett and I. M. Isaacs, On groups of central type, Math. Z. 179 (1982), 555-569.

7. I. M. Isaacs, Character correspondences in solvable groups, Advances in Math. 43 (1982), 284-306.

8. I. M. Isaacs, Blocks with just two irreducible Brauer characters in solvable groups, J. Algebra 170 (1994), 487-503.

9. G. Karpilovsky, Projective representations of finite groups, Monographs and Textbooks in Pure and Applied Mathematics 94. (Marcel Dekker, 1985).

10. R. A. Liebler and J. E. Yellen, In search of nonsolvable groups of central type, Pacific J. Math. 82 (1979), 485-492.

11. N. G. Ng, Degrees of irreducible projective representations of finite groups, J. London Math. Soc. (2) 10 (1975), 379-384.

Department of Mathematics

UNIVERSITY COLLEGE

DUBLIN

IRELAND 